\title{
ESTUDO DA VARIABILIDADE ESPACIAL DA CONDUTIVIDADE HIDRÁULICA DO ENTORNO DA LAGOA DA CONFUSÃO - TO
}

\author{
Pericles Souza Lima ${ }^{(\mathrm{a})}$;Fernando de Morais ${ }^{(\mathrm{b})}$; Letícia Giuliana Paschoal ${ }^{(\mathrm{c})}$ \\ ${ }^{(a)}$ Curso de Geografia, Universidade Federal de Tocantins, Campus de Poto Nacional. E-mail: \\ periclessouzalima22@gmail.com \\ (b) Programa de Pós-Graduação em Geografia, Universidade Federal de Tocantins, Campus de Poto Nacional. E-mail: \\ morais@uft.edu.br \\ (c) Programa de Pós-Graduação em Geografia, Universidade Federal de Tocantins, Campus de Poto Nacional. E-mail: \\ leticiagiulinapaschoal@gmail.com
}

\section{EIXO: BACIAS HIDROGRÁFICAS E RECURSOS HIDRICOS: ANÁLISE, PLANEJAMENTO E GESTÃO}

\begin{abstract}
Resumo
A escassez de água vem se tornando cada vez mais recorrente. Diante desse cenário, estudos visando alternativas para a conservação dos recursos hídricos têm se intensificado. O estudo da condutividade hidráulica se consolidou como instrumento importante nas investigações hidrológicas e geomorfológicas, por apresentar dados que influenciam no planejamento de uso do solo, análises denudacionais, estudo da velocidade de recarga dos aquíferos, entre outros. Desse modo, o presente trabalho visou apresentar a velocidade da Condutividade Hidráulica Satura $(K f s)$ no processo de recarga dos aquíferos no entono da Lagoa da Confusão - TO, relacionando os diversos aspectos envolventes na infiltração e percolação da água no solo. Para tal foram feitos ensaios de condutividade hidráulica saturada utilizando-se o permeâmetro Guelph. Os resultados indicaram certa homogeneidadenos fluxos,não se diferenciando em mais que duas ordens de grandeza, e mostraram que a área possui uma condutividade média.
\end{abstract}

Palavras chave: Água;Condutividade hidraulica saturada;Infiltração.

\section{Introdução}

Dada sua importância em apresentar dados que influenciam no planejamento de uso do solo, análises denudacionais, estudo da velocidade de recarga dos aquíferos, bem como contribuição para criação de melhores soluções para a reestruturação de aterros sanitários, análise de ambientes para implantação de cemitérios (CARNEIRO, 2008) entre outros aspectos (MAHLER e AGUIAR, 2001; BRANDT, 2010; FIORI; CAMPOS; ALMEIDA, 2010; BETIM 2013), a Condutividade Hidráulica (K) ou permeabilidade que pode ser definida como a facilidade com que o solo tem de transportar um fluido(GUIMARÃES; VIEIRA; FERNANDES, 2002; MESQUITA; MORAES, 2004; PRESS et al., 2006; BETIM, 2013), há tempos tem se tornando objeto de estudo de muitos pesquisadores.

Os estudos das propriedades hídricas do solo são de grande importância para a modelagem dos fluxos tanto na zona saturada, quanto na estimativa de recarga de aquíferos, mormente na área de grande 
expansão das atividades agrícolas, onde a demanda pelos recursos hídricos é maior, devendo, portanto, ser mais planejada. Com uso intensivo de defensivos agrícolas nas áreas de lavoura na planície do Médio Araguaia, a importância do entendimento da variabilidade espacial desta propriedade do solo ganha ainda mais notoriedade, constituindo-se uma variável significativa a ser levada em conta quando do planejamento e gestão ambientais para esta porção ecotonal da bacia hidrográfica do rio Araguaia, com características de wetland.

Dentre diversos fatores, a condutividade hidráulica é dependente dos atributos físicos dos solos, da morfologia destes, da viscosidade do fluido, da declividade do terreno, das condições atmosféricas, dascaracterísticas biológicas do solo e da ação antrópica(MORAIS, 2012; BETIM, 2013). Os estudos de condutividadehidráulicasaturada, do inglês (Field-saturatedhydraulicconductivity $-K f s$ ) têm sido realizadoscom o auxílio de alguns aparelhos, seja de laboratório ou de campo, dentre os quais podem ser destacados: ensaios de carga constante ou carga variável, infiltrômetros, ensaios de anéis concêntricos e os de permeâmetros de furo.

Os dados desta pesquisa foram coletados in situ utilizando o permeâmetro Guelph modelo IAC (Instituto Agronômico de Campinas). O permeâmetro Guelph é um equipamento de carga constante que mede a Condutividade Hidráulica (K) acima do lençol freático, sua composição consiste em um tubo de Mariotte que regula a água dentro do orifício (poço), um tubo de acrílico onde se coloca a água e um tripé de sustentação do equipamento. $\mathrm{O}$ aparelho tem sidobastante recomendado por ser prático, permitir rápida realização dos ensaios, baixo custo e baixoconsumo de água (GUIMARÃES; VIEIRA; FERNANDES, 2002; MORAIS, 2007;SOTO; CHANG; VILAR, 2009; BETIM, 2013).

Esse trabalho tem como objetivo apresentar a velocidade da Condutividade Hidráulica Saturada $(K f s)$ no processo de recarga do aquífero no entono da Lagoa da Confusão - TO, relacionando os diversos aspectos envolventes no procedimento de infiltração e percolação da água no solo e de sua movimentação verticalizada e horizontalizada (K), enfatizando a correlaçãoentre os valores de $(K f s)$ e os aspectos pedológicos da área.

Este estudo visa contribuir com um melhor entendimento do sistema em questão, além de fornecer parâmetros para um diagnóstico da vulnerabilidade dos recursos hídricos subterrâneos da área da bacia, podendo ainda ser utilizado durante a elaboração de programas de planejamento e gestão ambientas.A realização do trabalho se justifica pelo significativo avanço da agricultura sobre a paisagem de wetlands da zona de transição Cerrado/Floresta Amazônica, sendo imperiosa a abordagem da vulnerabilidade dos recursos hídricos sob a ótica da modelagem sistêmica na qual se baseia esta pesquisa. 


\section{2. Área de Estudo}

28 de Junho à 02 de Julho de 2017

A pesquisa foi realizada na bacia hidrográfica do córrego Barreiro, inseridano município de Lagoa da Confusão - TO, a uma distancia de 206 km de Palmas, capital do estado do Tocantins, via TO-255 e BR-226. Essa bacia situa-se entre as coordenadas geográficas: Lat: 1052'12.41”S, Long: 49³9'39.96”O e Lat: $10^{\circ} 44^{\prime} 01.34$ 'S, Long: 49³3'42.92”O e possui altitudes que variam de 170 a $230 \mathrm{~m}$.

A área (Figura 1) é objeto de estudo de um projeto mais amplo, intitulado: "Estudo da vulnerabilidade dos recursos hídricos subterrâneos na região de Lagoa da Confusão - Tocantins". Localizada na Bacia Sedimentar do Bananal, a Lagoa da Confusão está inserida em uma região de planície fluvio-lacustre fazendo parte do relevo cárstico da Formação Couto Magalhães (GORAYEB, 2011;PEREIRA; MORAIS, 2012).

A área de estudo possui clima úmido com moderada deficiência hídrica no inverno, evapotranspiração potencial apresentando variação média anual entre 1.400 e 1.700 mm, distribuindo-se no verão em torno de 390 e $480 \mathrm{~mm}$ ao longo dos três meses consecutivos com temperatura mais elevada. A precipitação média anual vai de $1.900 \mathrm{~mm}$ a $2.000 \mathrm{~mm}$ e a temperatura média anual chega aos $25^{\circ} \mathrm{C}$, o bioma predominante é o cerrado e o solo é do tipo Plintossolos (SEPLAN, 2012).

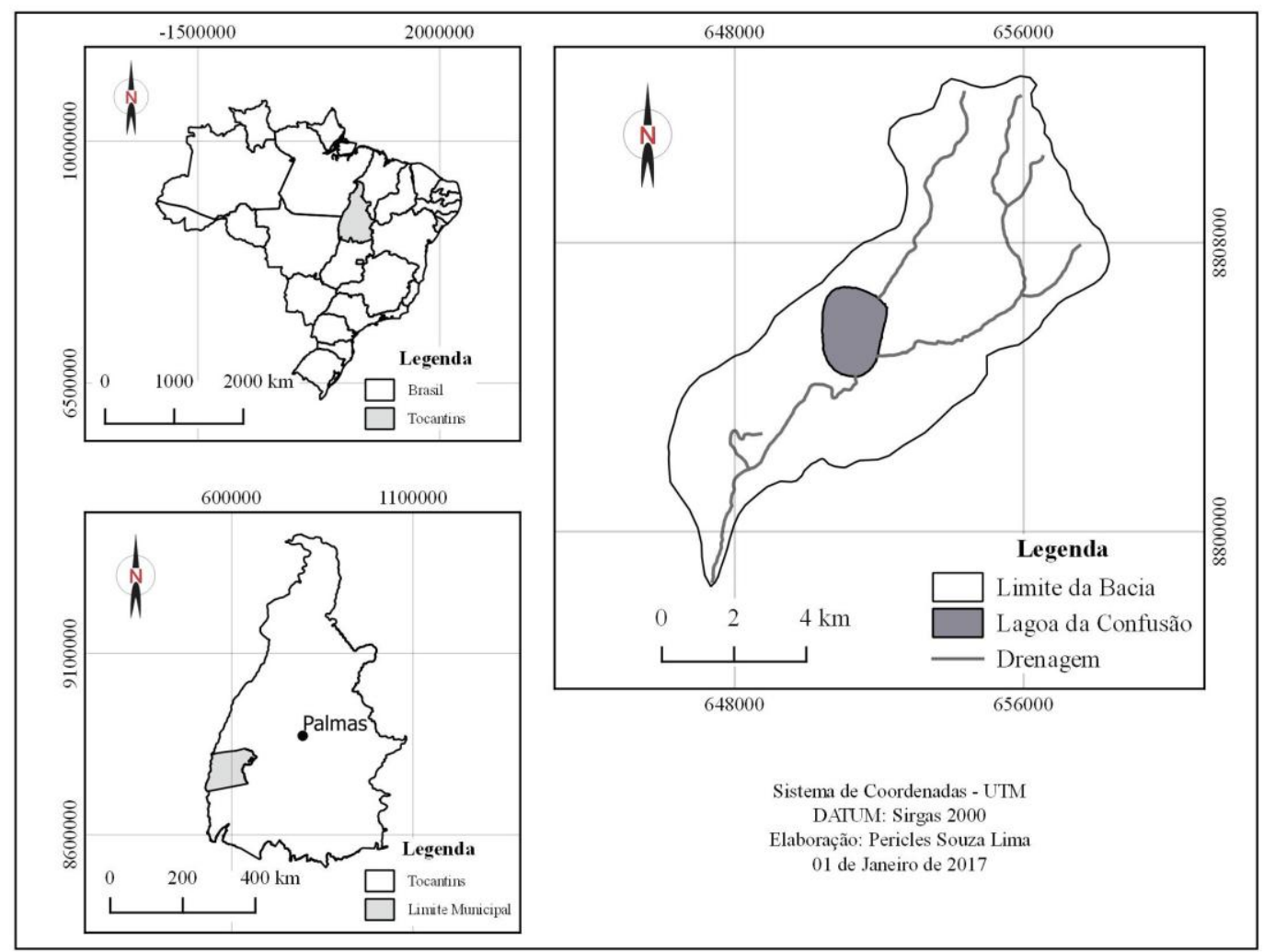

Figura 1 - Localização da área deestudo. 
XVII Simpósio Brasileiro

de Geografia Fisica Aplicada

I Congresso Nacional

de Geografia Física
OS DESAFIOS DA GEOGRAFIA FÍSICA NA FRONTEIRA DO CONHECIMENTO

Instituto de Geociências - Unicamp

Campinas - SP

28 de Junho à 02 de Julho de 2017

\section{Material e Metodologia}

Inicialmente, foramrealizados levantamentos bibliográficos que abordassem o estudo da condutividade hidráulica, levando em consideração tanto os conceitos e aplicabilidade deste tipo de pesquisa, como também os métodos mais viáveis para a realização da mesma. Posteriormente foram pesquisados materiais cartográficos sobre a área, buscando informações na carta topográfica: FolhaSC.22-Z-A-VI / Lagoa da Confusão, imagens de satélites, além textos de pesquisas já realizadas na região.

Tendo adquirido estas informações, foram realizadas visitas mais detalhadaspara o reconhecimento da área. A realização dos ensaiosin loco, ocorreram entre os dias 26 e 27 de outubro de 2016. Foram utilizados vários equipamentos para a concretização dos ensaios sendo eles: trado holandês e escarificador em nylon, respectivamente para abertura e preparo dos orifícios, recipiente para o armazenamento de água, trena, equipamento GPS, permeâmetro Guelph modelo (IAC) (Figura 2) e caderneta de campo.

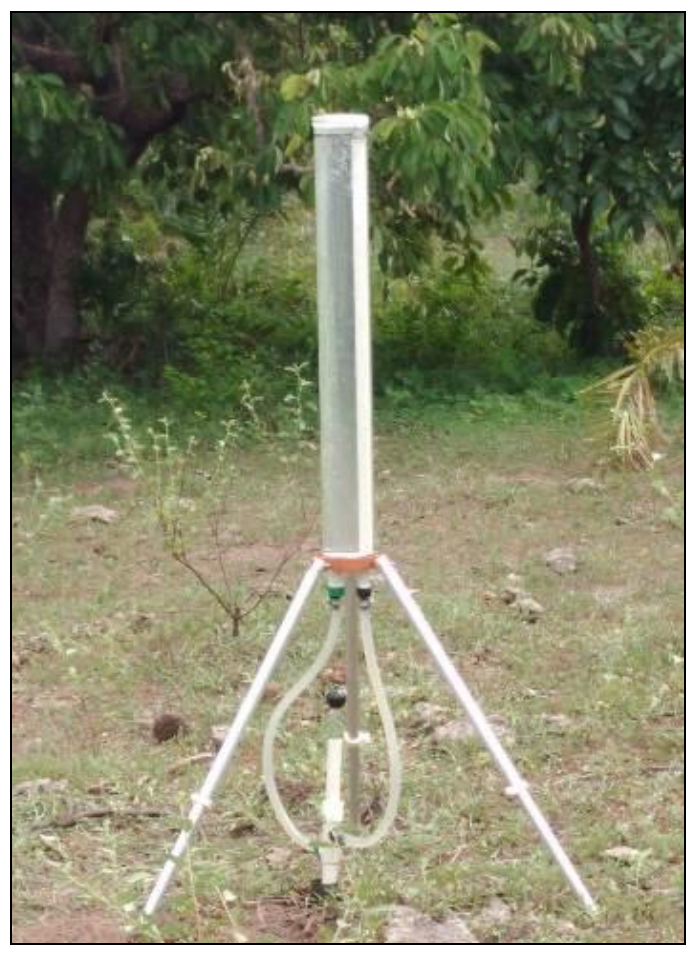

Figura 2 - Permeâmetro Guelph modelo (IAC)

Analisando o ambiente da bacia do córrego Barreiro, foram selecionados 12 pontos, onde se realizaram os ensaios de $K f$ s. Estes pontos foram escolhidos de maneira a abarcar por completo o entorno da Lagoa da Confusão. Os orifícios em subsuperfície, abertos com a ajuda de um trado, foram trabalhados cuidadosamentesendo bem escarificados para que as paredes dos próprios não danificassem e 
comprometessem a qualidade dos dados. Os ensaios de condutividade foram realizados a uma carga hidráulica $(\mathrm{H})$ de 10 cmem orifícios comprofundidade $(\mathrm{P})$ de $30 \mathrm{~cm}$.

Após a realização dos ensaios, os dados foram levados ao Laboratório de Análises GeoAmbientais (LGA) da Universidade Federal do Tocantins,para organização e análise.Com uso do software Excel trabalhou-se os valores adquiridos em campo, utilizando-seo coeficiente de Hazen (MORAIS, 2007), que leva em consideração os fatores granulométricos e a relação de vazios do meio físico, calculouse a condutividade hidráulica conforme a equação seguinte:

$$
K=\frac{C \cdot Q}{\left[2 \pi H^{2}+C \pi \alpha^{2}+\left(\frac{2 \pi H}{\alpha}\right)\right]}
$$

Onde:

K: condutividade hidráulica saturada;

Q: vazão em m/s;

C: coeficiente de Hazen;

a: raio do poço (furo);

$\alpha$ : coeficiente estipulado a partir dascaracterísticas do meio poroso, sobretudo a estrutura e a textura dos solos ensaiados.

A determinação dos valores de $\alpha$ se deu com base em Elricket al.(1989), que sugerem o valor de $\alpha$

$=12 \mathrm{~m}^{-1}$ para solos estruturados argilosos e siltosos, e também para areias finas e solos razoavelmente estruturados, como os encontrados na área de estudo.

A partir de então foi produzido um mapa temático de interpolação no softwareQGis(versão 2.14.3), apresentando a variação da velocidade do fluxo no solo do entorno da Lagoa da Confusão TO.Por exigir pouco tempo para a geração dos resultados e pela fidedignidade aos valores (LANDIM, 2000), o algoritmo utilizado para a interpolação dos dados foi o Inverso Ponderado da Distância.

\section{Resultados e Discussões}

Como apontam Mesquita e Moraes(2004),Ribeiro et al.(2007) e Morais (2012), ainfiltração e acondutividade hidráulicasaturada e não saturada são dependentesdos fatores físicos do solo: textura, estrutura, consistência dos agregados, porosidade eencrostamento superficial,que podem influenciar 


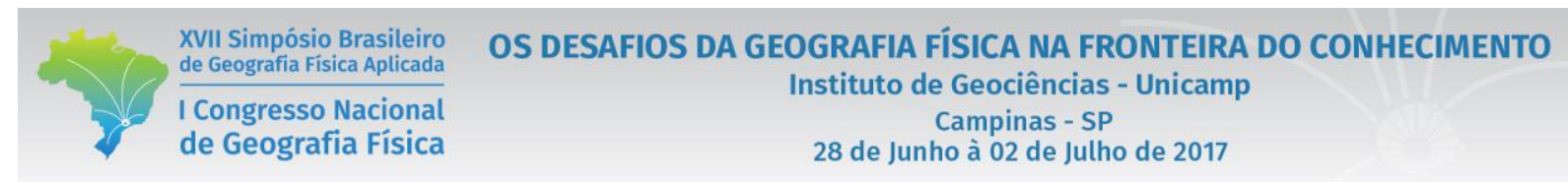

noprocesso inicial deentrada de água no solo. Logo a mesma também depende das propriedades do material percolante e das características do relevo.

Diante dos investimentos no setor agrícola da região, a área estudada apresenta-se bastante modificada pela ação antrópica, tanto na zona urbana, quanto na rural. Assim, além da alteração do uso do solo, agora destinado à agricultura, essa situação tem contribuído também com o aumento do número de loteamentos e, consequentemente, da malha urbana, fatos que influenciam na infiltração e na condutividade hidráulica (BATISTA; SOUZA, 2015).

Os ensaios de $K f s$ apresentaram valores com ordem de grandeza de $10^{-3}$ e de $10^{-4} \mathrm{~m} / \mathrm{s}$. Após o preparo dos equipamentos os experimentos foram feitascom uma média de 10 a 12 minutos de leituras para cada local. Os resultados finais obtidos para o entorno da Lagoa da Confusão foram interpolados e representados de maneira espacializada na bacia do córrego Barreiro (Figura3).

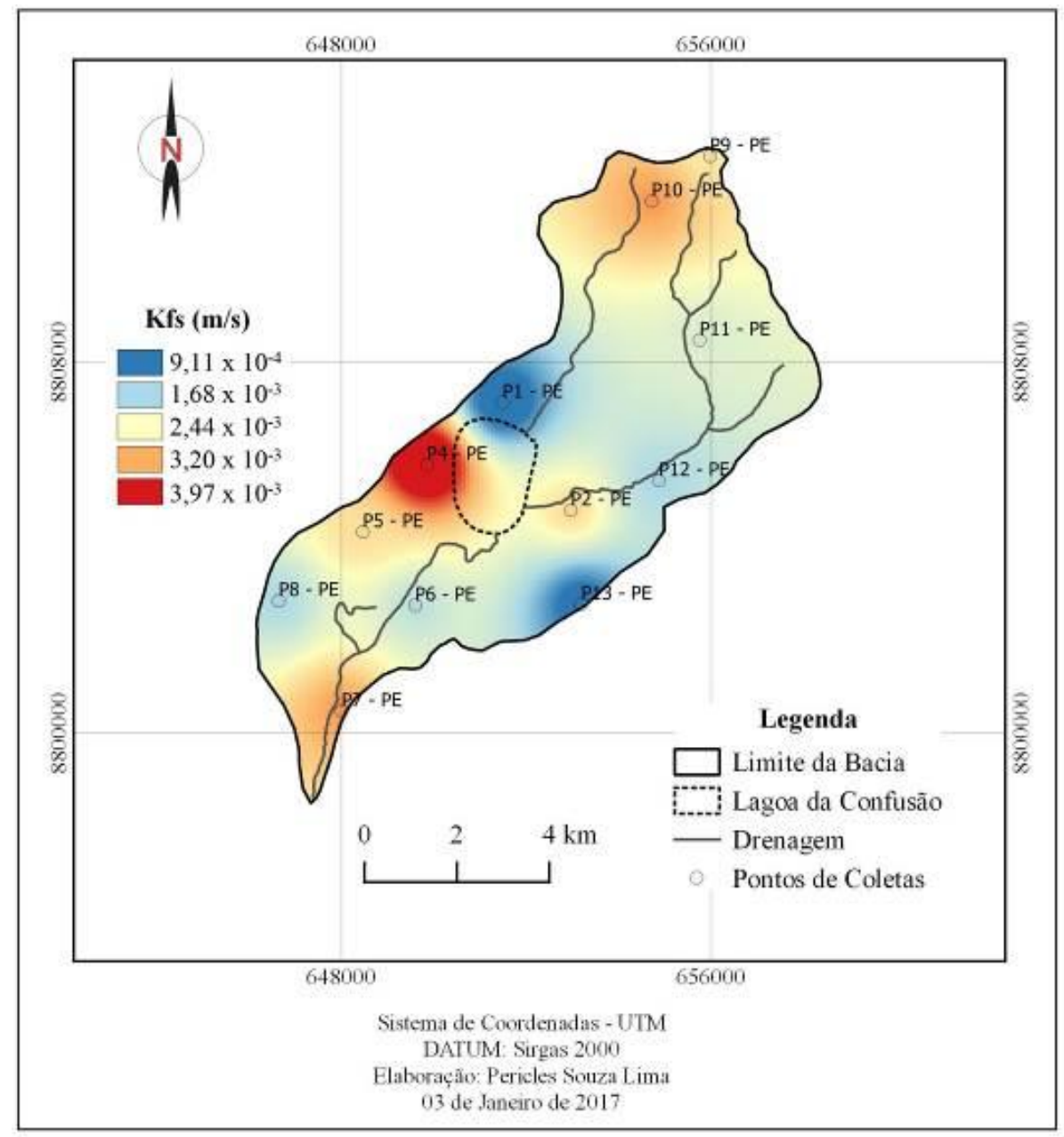

Figura 3 - Classificação espacial da variabilidade da Condutividade Hidráulica Saturada do entorno da Lagoa da Confusão - TO. 
A partir da distribuição interpolada, como visto na figura 3, é possível afirmar que os fluxos do entorno da Lagoa da Confusão possuem certa uniformidade, apresentando valores de $K f s$ médios em praticamente toda área estudada,o que permite averiguar que o processo de recarga do freáticoé rápido. Por meio da interpretação desses dados, pode-se também inferir que a vulnerabilidade à contaminaçãodo lençol freático tende a ser de média a elevada, principalmente pelosignificativo uso de defensivos agrícolas, que ao entrar em contato com a água da chuva, acabam infiltrando nos solos, podendo afetar osaquíferos que não são muito profundos na área de estudo.

Três pontos na bacia hidrográfica estudada destacam-se: os pontos P1-PE, P4-PE situados na zona urbana, e o ponto P13-PE, na zona rural.Os pontos P1-PE e P13-PE, embora localizados em áreas com usos da terra diferentes, apresentaram os menores valores de $K f s$, na ordem de grandeza $9,11 \times 10^{-4}$. Já o ponto P4-PE foi o que teve maior valor de $K f s 3,97 \times 10^{-3}$. Mesmo se diferenciando pela tonalidade de cor forte, o ponto P4-PE nãodestoa da ordem de grandeza $10^{-3}$, na qual, com exceção dos pontos P1-PE e P13PE, situam-setodos os pontos restantes, o que significa que o fluxo de infiltração tende a apresentar pouca variação por toda a área de estudo.

A $K f s$ do entorno da Lagoa da Confusão pode ser relacionada aosatributos estruturais. Considerando os estudos de (RESENDE et al.,2007) que apontam a organização das partículas primárias do solo como de grande importância para a configuração do espaço poroso e para a percolação, foi notado que os pontos P2-PE, P4-PE, P10-PE e P11-PE, tem estruturação do tipo granular, contendo ainda a presença de cascalhos.

Os pontos P8-PE e P9-PE também apresentam estruturações semelhantes, além de apresentarem influência de bioporos. Os pontos P1-PE e P13-PEos quais tiveram menor condutividade não se mostraram bem estruturados. A declividade não foi fator tão condicionante na variabilidade da ( $K f s)$, haja vista que o relevo é bastante suavizado.

\section{Considerações Finais}

Diante dos resultados, compreende-se que o entorno da Lagoa da Confusão possui pouca variabilidade da Condutividade Hidráulica Saturada.A uniformidade nas taxas dos fluxos no solo aponta para o fato de que a água percolantegasta um período equiparado por toda a áreaaté chegar à zona de saturação. Os valores não se diferenciam muitopor ordem de grandeza, e mesmo os dados que maisdestoamaindaapresentam valores próximos da maioria dos pontos amostrados. 
Os valores de $K f s$ permitem afirmar que a capacidade de recarga é similar em toda área da bacia. Mesmo tendo conhecimento da influencia do material percolante na movimentação no meio físico, é destaque inferir que esta homogeneidade na recarga possa ser relevante ao se tratar de possíveis impactos ocasionados por poluentes, provenientes das instalações no meio urbano e dos defensivos usados nas lavouras.O permeâmetro Guelph modelo (IAC) se mostrou eficiente, conforme o esperado, tendo uma média de gasto de água de pouco mais de 10 litros por ensaio, além de ser de fácil transporte.

\section{Agradecimentos}

A presente pesquisa foi desenvolvida com auxílio financeiro da Fundação de Amparo à Pesquisa do Estado do Tocantins, através do Programa de Pesquisa em Recursos Hídricos, processo $\mathrm{n}^{\circ}$ 2014.20300.000009. O estudo foi também financiado pelo Conselho Nacional de Desenvolvimento Científico e Tecnológico - CNPq através do Edital Universal, processo nº 485433/2013-3.

\section{Referências Bibliográficas}

BATISTA, D. F; SOUZA, F. A de.Avaliação da condutividade hidráulica do solo sobre condições de cobertura por cerrado e pastagem. In: REVISTA ELETRÔNICA DO CURSO DE GEOGRAFIA - UFG/REJ,Jataí-GO. n.25, p. 1-20, 2015.

BETIM, L. S. Caracterização da condutividade hidráulica dos solos e estudo da vulnerabilidade à contaminação dos aquíferos da sub-bacia do córrego Palmital. 2013. 186 f. Dissertação (Mestrado em Engenharia Civil) - Departamento de Engenharia Civil, Universidade Federal de Viçosa. Viçosa.

BRANDT, M. A. C.Mapeamento da condutividade hidráulica da cobertura do aquífero guarani, situado na bacia hidrográfica dos rios Jacaré-guaçú e Jacaré-pepira, na porção centro-norte do estado de São Paulo.2010. 36 f. Trabalho de Conclusão de Curso (Graduação em Engenharia Ambiental) - Instituto de Geociências e Ciências Exatas, Universidade Estadual Paulista. Rio Claro.

CARNEIRO, V.S. Impactos causados por necrochorume de cemitérios: meio ambiente e saúde pública. In: XV CONGRESSO BRASILEIRO DE ÁGUAS SUBTERRÂNEAS; Natal; Rio Grande do Norte. P. 1-18, 2008.

ELRICK, D. E; REYNOLDS, W. D; TAN, K.A. 1989. Hydraulic conductivity measurements in the unsaturated zone using improved well analyses. Ground Water Monitoring Review, 9: 184-193.

FIORI, J. P de O; CAMPOS, J. E. G; ALMEIDA, L. Variabilidade da condutividade hidráulica das principais classes de solos do estado de Goiás.São Paulo, UNESP, Geociências, v. 29, n. 2, p. 229-235, 2010.

GORAYEB, P. S de S. Geologia do estado do Tocantins: aspectos gerais e conhecimento atual. In: MORAIS, F de. (Org).Contribuição a geografia física do estado do Tocantins. Goiânia: Kelps, 2011. p. 19-46.

GUIMARÃES, M. da S. B; VIEIRA, B. C; FERNANDES, N. F. Comparação da condutividade hidráulica saturada medida através de métodos de campo e de laboratório.In: $10^{\circ}$ CONGRESSO BRASILEIRO DE GEOLOGIA DE ENGENHARIA E AMBIENTAL. Ouro Preto-MG.p. 1-12, 2002. Código: 099IG.

LANDIM, P.M.B. Introdução aos métodos de estimação espacial para confecção de mapas. DGA,IGCE,UNESP/Rio Claro, Lab. Geomatemática,Texto Didático 02, 20 pp. 2000. Disponível em $<$ http://www.rc.unesp.br/igce/aplicada/textodi.html>. 
MAHLER, C. F; AGUIAR, A. B de. Determinação da permeabilidade in situ em aterros de resíduos sólidos. In: $21^{\circ}$ CONGRESSO BRASILEIRO DE ENGENHARIA SANITÁRIA E AMBIENTAL. ABES - Trabalhos Técnicos. p. 1-9, 2001.

MESQUITA, M. da G. B. de F; MORAES,S. O. A dependência entre a condutividade hidráulica saturada e atributos físicos do solo.Santa Maria,Ciência Rural, v.34, n.3, p.963-969, 2004.

MORAIS, F. Estudo dos fatores pedogeomorfológicos intervenientes na infiltração em zonas de recarga no complexo metamórfico bação. 2007. 132 f. Tese (Doutorado em Evolução Crustal e Recursos Naturais) Departamento de Geologia, Universidade Federal de Ouro Preto.Ouro Preto.

MORAIS, F. Infiltração - uma variável geomorfológica. Caderno de Geografia, v.22, n.38, 2012.

PEREIRA, G. C; MORAIS, F. Geofísica aplicada ao estudo dos fluxos subsuperficiais no entorno da Lagoa da Confusão - TO. REVISTA GEONORTE, Edição Especial, v.2, n.4, p.1475 - 1483,2012.

PRESS, F; SIEVER, R; GROTZINGER, J; JORDAN, T. H. Para Entender a Terra. $4^{a}$ ed. Porto Alegre: Bookman, 2006. 656p.

RESENDE, M; CURI, N; RESENDE, S. B de; CORRÊA, G. F. Pedologia: base para distinção de ambientes. $5^{\text {a }}$ ed. Lavras: Editora UFLA, 2007.

RIBEIRO, K. D; MENESES, S. M; MESQUITA, M da .G .B de. F; SAMPAIO, F de. M. T.Propriedades físicas do solo, influenciadas pela distribuição de poros, de seis classes de solos da região de Lavras-MG.Ciênc. agrotec. Lavras, v. 31, n. 4, p. 1167-1175, jul./ago 2007.

SEPLANSecretaria do Planejamento e da Modernização da Gestão Pública, Diretoria de Zoneamento Ecológico-Econômico - DZE. 6 ${ }^{a}$ ed.Palmas: Seplan, 2012.

SOTO, M. A; CHANG, K. H; VILAR, O. M. Análise do método do permeâmetro Guelph na determinação da condutividade hidráulica saturada. Águas Subterrâneas, v.23, n.01, p.137-152, 2009. 